\title{
Study on characteristics of resonant frequency of wireless power transmission based on PCB coil
}

\author{
Yanping Cong ${ }^{1, a}$, Xiuwei Wang ${ }^{2, b}$ \\ ${ }^{1}$ Department of Computer Science and Technology, Ocean University of China, Qingdao, 266100, \\ China \\ ${ }^{2}$ Department of Computer Science and Technology, Ocean University of China, Qingdao, 266100, \\ China \\ acongyp@ouc.edu.cn, b17864270587@sina.cn
}

Keywords: Wireless energy transfer, PCB coil, the resonant frequency, the number of turns, edge spacing

\begin{abstract}
With the development of science and technology, the application of implantable biomedical electronic devices which play an important role in biological research and medical diagnostics becomes increasingly popular[1]. Nowadays wireless energy transfer is one of the most popular research field, it can penetrate the skin to supply electric energy to implantable biomedical electronic devices steady, so it is more and more used in the biomedical field. The main constraints restrict for wireless charging technology to realize miniaturization and portability is that the volume of the transmission coil is difficult to reduce. The production process of the PCB(printed circuit board) coil is simple, and its width, thickness, line spacing and other parameters can be precisely controlled, mass production of PCB coils have same parameters, meanwhile ,PCB coil is easy to integrate with external circuit. In order to improve the efficiency of the radio transmission system fundamentally, by comparing the test methods, we study the PCB coil width and wire spacing, analyze the impact of these two factors on the resonant frequency. And we reach the following conclusion: The resonant frequency is negative correlated to the number of turns when the wire spacing is fixed. When the number of turns is constant, the wire spacing of the coil and the resonant frequency are positively correlated.
\end{abstract}

\section{Introduction}

Magnetic coupled resonant wireless power transmission technology has the advantages of high transmission distance and high transmission efficiency, and it is a new type of energy transmission technology. Application prospect of the technology is very broad, not only in electric vehicle charging system, wireless sensor network and RFID, get extensive application, operating in household appliances, small mobile tools, traffic tools, industrial robots, oil and mine, water, medical, aerospace and other fields also have great application value and for charging technology and the development of electromagnetic theory also has very important significance[2]. At the same time, one of the frontiers in the field of magnetic coupling resonant wireless power transmission technology is the wireless transmission field, many domestic and foreign scientists are working on high security, high efficiency, long distance, high power wireless energy transmission device[3]. The resonant frequency is a very important index of the magnetic resonance wireless energy transmission technology[4]. However, the theoretical research on the technology is still not perfect, there are still a lack of experimental research, from the overall point of view the technology is still in its infancy, to practical, but also need to solve many problems. If in the process of transmission, encountered outside magnetic material will affect the coil inductance changes, resulting in changes in the resonant frequency of the system, that is, the detuning[5][6], causing the sharp decrease of the transmission efficiency, also working environment temperature change and receive side load changes are likely to lead to detuning. In this paper, the resonance frequency is analyzed from the angle of the change of the transmission performance of the system from the line spacing and line width variation. 
Because the wire thickness of the coil is much smaller than the line width, the PCB coil can ignore the influence caused by the proximity effect, and the thickness of the wire is very small. The large AC resistance is the main reason why the transmission efficiency is hard to improve[7][8]. PCB coils are rectangular, circular and rectangular, and so on. The resonant system has a frequency division in the near distance transmission, so that the efficiency is reduced. In order to solve this problem, this paper analyzes the influence of the number of turns and the edge spacing on the resonant frequency, and determines the main factors that affect the resonant frequency.

\section{Coil characteristics influence}

Resonant frequency of the system is in the resonance state signal of the driving frequency, coil and the inherent frequency is not with a frequency, but the resonant frequency by the inherent frequency of the coil decision. Theoretically, when the signal driving frequency is equal to the coil's inherent frequency that system to achieve resonance[9], but the results is not the case, when the two ends of the receiving coil voltage reaches a maximum, that is the system to reach resonance, and this time the signal driving frequency is slightly larger than the natural frequency of the coil. The following is a different number of turns of the coil in the simulation of winding way. This is because there is between the two coils coupled (mutual inductance $M$ should satisfy: $M-\mathrm{k} \bullet \sqrt{L_{1} L_{2}}$ ) [10],the equivalent of the resonant inductor and capacitance values change, thus changing the resonant frequency see Figure 1 (a) and (b).

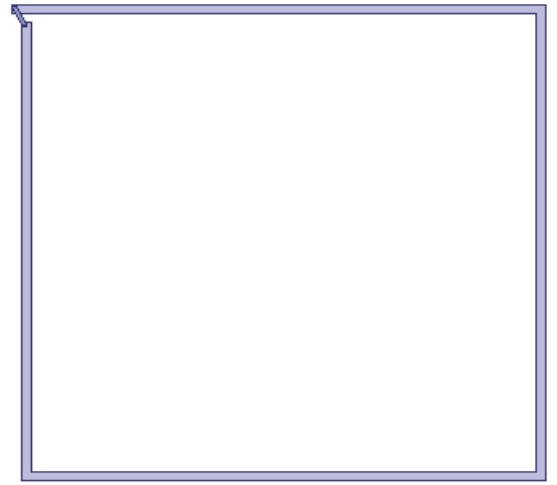

(a) Plane rectangular single turn coil winding method

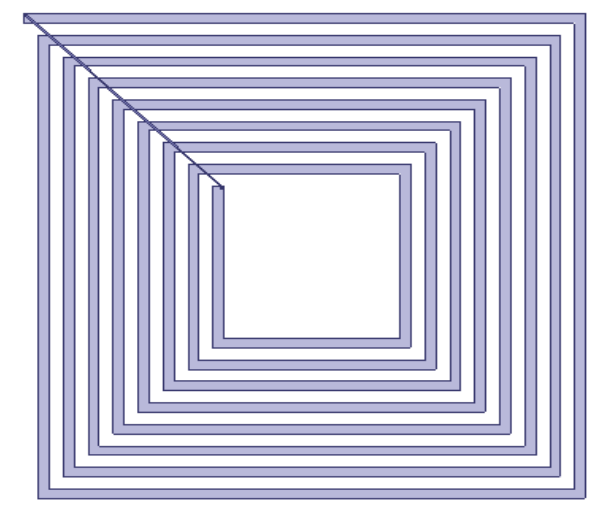

(b) Flat rectangular coil winding method

Fig.1. Coil winding method

In this paper, the PCB coil material is copper, PCB board material is FR4_epoxy, Table 1 shows all the electrical parameters. In Figure 2, the length and width of the coil and the distance between the coil and the coil are shown,and the physical parameters of the coil are represented by the following symbols: $\mathrm{L}$ is the length of the coil, $\mathrm{W}$ represents the width of copper, S represents the distance between adjacent coils margin line, h_c represents copper thickness (In this paper, the thickness of copper is all $0.035 \mathrm{~mm}$ ), $\mathrm{h}$ _f represents the thickness of the substrate FR4_epoxy (In this paper, the thickness of PCB plate is all $0.33 \mathrm{~mm}$ ), $\mathrm{N}$ represents the total number of turns of the coil.

Table 1 PCB parameter setting of rectangular coil

\begin{tabular}{|c|c|}
\hline Electrical parameter & Set value \\
\hline N & $2 \sim 7$ turns \\
S & $40-80 \mathrm{mil}$ \\
L & $4 \mathrm{~cm}$ \\
W & $30 \mathrm{mil}$ \\
h_f & $0.33 \mathrm{~mm}$ \\
h_c & $0.035 \mathrm{~mm}$ \\
\hline
\end{tabular}




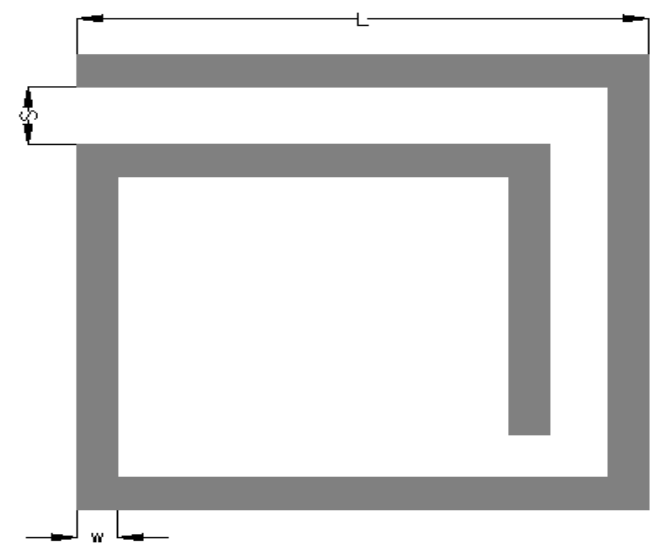

Fig.2. Labeling coil parameters

\section{Side coil spacing and turns parameters}

Through the simulation analysis, this paper has obtained the coil side spacing and coil turns contrast data, and the simulation of the data of the list analysis, draw the conclusion that the influence of the edge distance and coil turns on the resonant frequency.

Table 2 Effects of different coil turns and edge spacing on the resonance frequency

\begin{tabular}{|c|l|l|l|l|l|l|l|l|l|}
\hline $\mathrm{n}$ & \multicolumn{1}{|c|}{40} & \multicolumn{1}{|c|}{45} & \multicolumn{1}{c|}{50} & 55 & \multicolumn{1}{c|}{60} & \multicolumn{1}{c|}{65} & \multicolumn{1}{c|}{70} & \multicolumn{1}{c|}{75} & \multicolumn{1}{c|}{80} \\
\hline 2 & 164.3 & 177.66 & 191.7 & 206.08 & 218.64 & 227.76 & 240.42 & 239.14 & 250.44 \\
\hline 3 & 115.9 & 127.44 & 138.88 & 147.48 & 156.44 & 159.54 & 165.88 & 172.74 & 181.98 \\
\hline 4 & 98.4 & 106.04 & 114.98 & 123.12 & 126.92 & 134.98 & 140.8 & 148.22 & 155.3 \\
\hline 5 & 84.96 & 94.64 & 102 & 109.76 & 113.98 & 120.42 & 127.84 & 135.2 & 141.98 \\
\hline 6 & 77.72 & 85.9 & 93.58 & 98.46 & 105.72 & 113.02 & 120.84 & 127.58 & 135.02 \\
\hline 7 & 58.68 & 74.72 & 76.78 & 83.4 & 92.88 & 98.88 & 108.86 & 117.06 & 131.98 \\
\hline
\end{tabular}

According to Table 2 data, we can see that when the number of turns of the coil and the linewidth is certain, edge spacing from 40mil to $80 \mathrm{mil}$, the resonant frequency of the coil is increasing gradually with the increase of edge spacing, the resonant frequency and edge spacing into proportional; when the coil edge spacing must, coil turn number increased from 2 to 7 , the resonant frequency of the coil decreases with the increase of the number of turns of the coil, namely resonance frequency and the coil surface area was negatively correlated.

\section{PCB rectangular coil parameters influence on the resonant frequency}

\section{PCB rectangular coil of wire effects on the resonant frequency of side space}

Through HFSS simulation software to the rectangular coil were several sets of simulation, and the simulation data is analysed and processed, Figure 3 edge spacing effect on the resonant frequency curves are obtained. The abscissa is edge spacing $\mathrm{S}$, ordinate is resonant frequency $\mathrm{F}$, set different edge spacing $S_{i}$ (where $i=1,2,3 \ldots$...), Corresponding to different resonant frequencies are $F_{i}$ (wherein $i=1,2,3 \ldots$. ), and the resonance frequency of the series resonant circuit is $f_{r}$, according to the resonant circuit and made analysis, it made the following formula:

$$
\begin{aligned}
& f_{1}=f_{r}-\frac{\mathrm{S}_{i} \bullet F_{i}}{2} \\
& f_{2}=f_{r}+\frac{S_{i} \bullet F_{i}}{2}
\end{aligned}
$$


Among them, $\mathrm{f}_{1}, \mathrm{f}_{2}$ are the corresponding resonant frequency of the different edge distance, which is further derived from the above formula, the relationship between the line spacing and the resonant frequency is obtained:

$$
f_{r}=\frac{2 \bullet S_{i}}{\sqrt{f_{1} \bullet f_{2}}}
$$

It can be known that the gap between the coil and the resonant frequency is positively correlated, that is, when the coil turns is certain, the increase of the side space of the coil will lead to the increase of the resonance frequency.

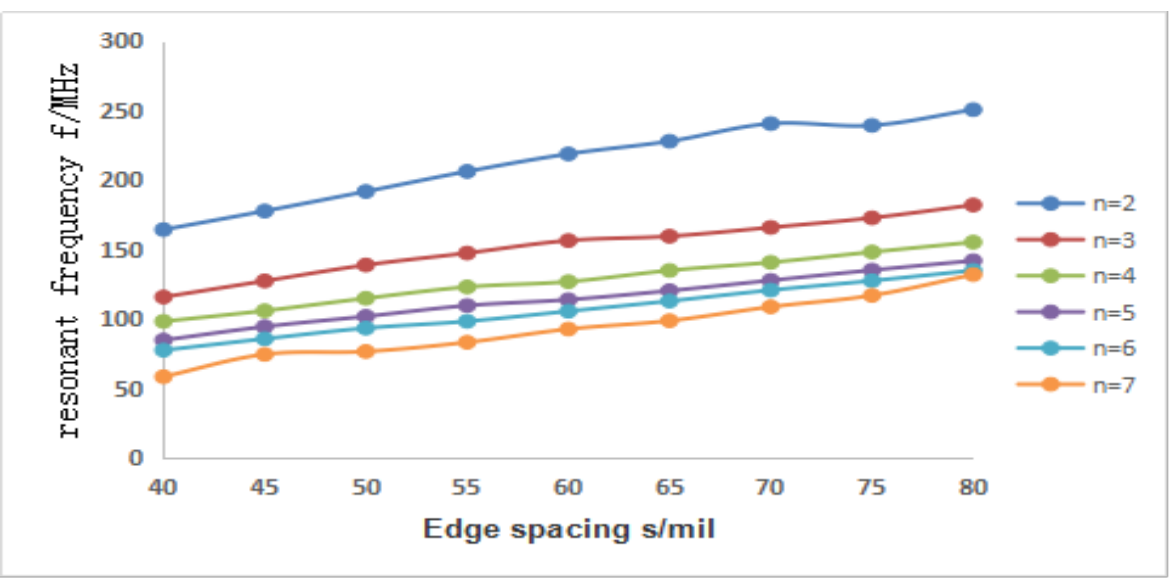

Fig.3. Influence of edge spacing on the resonance frequency of the coil

But with respect to the individual parameters, the increase in the side space of the coil leads to the increase of the resonance frequency, which is not so obvious. Conductor width can not increase too much, because the width of the wire will affect the distribution of magnetic field across the surface of the PCB board, therefore, the wire side spacing is one of the important factors that affect the resonant frequency.

\section{Effect of PCB rectangular coil resonant frequency linewidth}

Similarly, by HFSS rectangular coil were several sets of simulation, and the simulation data were processed and analysed, here set the distance between any two groups of different line spacing $S_{\mathrm{i}}$ corresponding to the resonant frequency is $f_{i}$, the different number of turns of the coil $N_{\mathrm{i}}$, different surface area: $A=2 *\left[\left(\mathrm{~L}+\left(\mathrm{L}-\left(N_{\mathrm{i}}-1\right) * S_{\mathrm{i}}\right)+\ldots ..\right) * W_{i}\right]$ (where $\left.\mathrm{i}=1,2,3 \ldots.\right)$, now the table by the ratio of the square of the length of area and coil resonant frequency, it is concluded that the as graph of Figure 4 , when the coils of line spacing is certain, resonant frequency with the increase of the number of turns of the coil gradually reduced. Studies have shown that the rate of change of the resonant frequency is $\eta=\frac{\left|f_{i}-f_{j}\right|}{W_{i} * S_{i}}$, after the verification and analysis of that curve variability $\eta$ than edge spacing of 40mil and coil edge spacing and width remained unchanged, with the increased number of turns in the coil, resonant frequency decreased gradually, after due to changing rates $\frac{\left|f_{i}-f_{j}\right|}{W_{i} * S_{i}}<0.01 \%$, the rate of change tends to 0 , i.e., changes in rate with increasing edge spacing significantly reduced. 


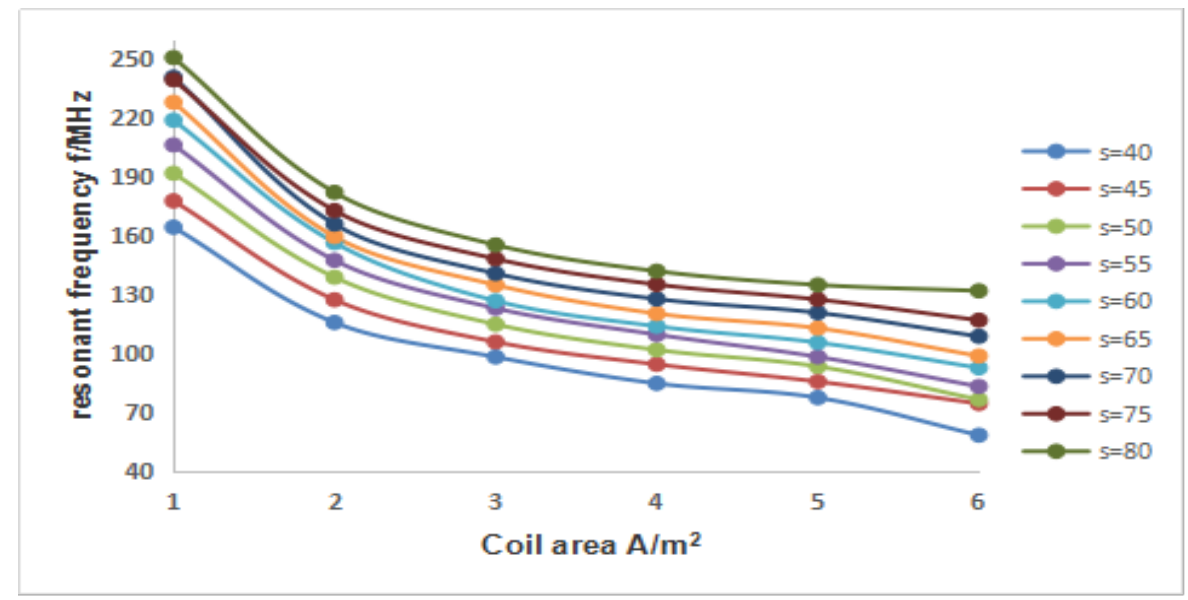

Fig.4. Influence of coil surface area of the coil's resonant frequency

\section{Summary}

In this paper, the radio transmission technology is briefly reviewed, and the calculation formula of resonant frequency is introduced, especially the influence of the electrical parameters of the coil section on the resonant frequency is analyzed in detail. The design of the coil is one of the key technologies of radio energy transmission. The high resonant frequency of the coil has great influence on the transmission performance of the system, including transmission distance, transmission efficiency, transmission power and so on. The effects of different resonant frequency on the efficiency of different linewidth and edge spacing are analyzed. Such as the coil parameters of the design, including the coil shape, turn, turn spacing, linewidth, material selection, etc., this paper on the use of HFSS simulation of the coil is also briefly described. Finally, we analyzed coil width and space of turns and influence on the resonant frequency, and drew the conclusion: The resonant frequency is negative correlated to the number of turns when the wire spacing is fixed. When the number of turns is constant, the wire spacing of the coil and the resonant frequency are positively correlated.

\section{Acknowledgements}

This paper received funding of China International Scientific and Technological Cooperation Special (2013DFA10490) and Qingdao innovation and entrepreneurship leading talent project (13-cx-2).

\section{References}

[1] Manolatou C, Khan M J, Fan Shanhui, et al. Coupling of modes analysis of resonant channel add-drop filters[J]. IEEE Journal of Quantum Electronics, 1999,35(9): 1322-1331

[2] Hirai J J, Kim T W, Kawamura A. Wireless transmission of power and information for cableless linear motor drive[J]. IEEE Transactions on Power Electronics, 2000, 15(1): 21-27.

[3] Shen Na,Li Changsheng,Zhang He.Modeling and analysis of wireless power transmission system based on magnetic coupling resonance[J].Chinese Journal of Scientific Instrument,2012(12): 2735-2 741.

[4] Li Yang,Yang Qingxin,Yan Zhuo,et al.Characteristic of frequency in wireless power transfer system via magnetic resonance coupling[J].Electric Machines and Control,2012(7):7-11

[5] M Ali, G R Dougal. A miniature packaged rectenna for wireless power transmission and date telemetry[C]. IEEE International Workshop on Antenna Technology Small Antennas and Novel Metamaterials, Mar. 2006:225-228. 
[6] Chwei-Sen Wang, Oskar H Stielau. Design considerations for a contactless electric vehicle battery charger[C]. IEEE Transactions on Industrial Electronics, 2005,52(5): 1308-1314.

[7] Darrin J Yong. Wireless powering and date telementry for biomedical implants[C]. The 31st Annual international Conference of the IEEE EMBS,Minnesota, USA, 2009: 3221-3224.

[8] Joaquin J Casanova, Zhen NingLow, Jenshan Lin.Design and optimization of a class-E amplifier for a loosely coupled plannar wireless power system[C].IEEE Transactions on Circuits and Systems, 2009,56(11): 830-834.

[9] Qingxin Yang, Haiyan Chen, Suzhen Liu, et al. Dynamic modeling of magnetic system constructedwith giant magneto strictive thin film using elementfree galerkin method[J]. IEEE Transactions on Magnetics, 2006, 42(4): 939-942.

[10] Marracci M, Tellini B,Zappacosta C,elal. Critical parame ters for mutual inductance between Rogowski coil and primary conductor[J]. IEEE Transactions on Instrumentation and Measurement, 2011,60(2):625-632. 